\title{
Divide and Conquer: EKF SLAM in $O(n)$
}

\author{
Lina M. Paz, Member, IEEE, Juan D. Tardós, Member, IEEE, and José Neira, Member, IEEE
}

\begin{abstract}
In this paper, we show that all processes associated with the move-sense-update cycle of extended Kalman filter (EKF) Simultaneous Localization and Mapping (SLAM) can be carried out in time linear with the number of map features. We describe Divide and Conquer SLAM, which is an EKF SLAM algorithm in which the computational complexity per step is reduced from $O\left(n^{2}\right)$ to $O(n)$, and the total cost of SLAM is reduced from $O\left(n^{3}\right)$ to $O\left(n^{2}\right)$. Unlike many current large-scale EKF SLAM techniques, this algorithm computes a solution without relying on approximations or simplifications (other than linearizations) to reduce computational complexity. Also, estimates and covariances are available when needed by data association without any further computation. Furthermore, as the method works most of the time in local maps, where angular errors remain small, the effect of linearization errors is limited. The resulting vehicle and map estimates are more precise than those obtained with standard EKF SLAM. The errors with respect to the true value are smaller, and the computed state covariance is consistent with the real error in the estimation. Both simulated experiments and the Victoria Park dataset are used to provide evidence of the advantages of this algorithm.
\end{abstract}

Index Terms-Computational complexity, consistency, linear time, precision, simultaneous localization and mapping (SLAM).

Manuscript received August 9, 2007; revised April 15, 2008. This work was supported in part by the European Union under Project RAWSEEDS FP6-IST045144 and in part by the Dirección General de Investigación of Spain under Project SLAM6DOF DPI2006-13578. Preliminary versions of this work were published in the Proceedings of the 2007 International Conference on Robotics and Automation, Rome, Italy, April 2007, pp. 1657-1663 and presented at the 2007 Robotics: Science and Systems Conference, Atlanta, GA, June 2007. This paper was recommended for publication by Associate Editor W. Burgard and Editor L. Parker upon evaluation of the reviewers' comments.

The authors are with the Instituto de Investigación en Ingeniería de Aragón, Universidad de Zaragoza, Zaragoza E-50018, Spain (e-mail: linapaz@unizar.es; tardos@unizar.es; jneira@unizar.es).

This paper has supplementary downloadable multimedia material available at http://ieeexplore.ieee.org. provided by the author. This material includes three AVI videos dcslam_loop.avi, dcslam_xvid_lawn.avi, and dcslam_ xvid_spiral.avi that show the execution of both EKF SLAM and D\&C SLAM for the same sample data using three simulated experiments: loop closing, lawn mowing, and outward spiral. The D\&C SLAM process is based on building a sequence of independent local maps that are represented with different colors. Local maps of the same size are joined by following a hierarchical maps structure. The frames have been time stamped so that the actual running times of the algorithms in our Matlab implementation are shown for comparison. The video dcslam_xvid_victoria.avi shows the execution of both EKF SLAM and D\&C SLAM for the Victoria Park data set. Each algorithm solves data association on its own. This allows seeing when the estimator falls out of consistency precisely because data association starts to fail; there are some minor differences due to missed associations in the case of EKF. In this experiment, the total cost of D\&C SLAM is one fifth of the total cost of standard EKF, (130.24s on a $2.8 \mathrm{GHz}$ Pentium IV, compared to 590.48s for EKF SLAM). The videos dcslam_xvid_loop.avi, dcslam_xvid_lawn.avi, dcslam_xvid_spiral.avi, and dcslam_xvid_victoria.avi, all in the file dcslam_videos.zip have been tested on: VLC media plater: http://www.videolan.org, and Windows Media Player with xvid codec from: http//www.xvid.org/. The size of the video is not available.Contact linapaz@unizar.es for further questions about this paper.

Color versions of one or more of the figures in this paper are available online at http://ieeexplore.org.

Digital Object Identifier 10.1109/TRO.2008.2004639

\section{INTRODUCTION}

$\mathbf{T}$ HE SIMULTANEOUS Localization and Mapping (SLAM) problem deals with the construction of a model of the environment being traversed with an onboard sensor, while at the same time maintaining an estimation of the sensor location within the model [3], [4]. Solving SLAM is not only central to the effort of conferring real autonomy to robots and vehicles, but also opens possibilities in applications where a sensor moves with 6 DOF, such as augmented reality. SLAM has been the subject of much attention since the seminal work in the late 1980s [5]-[8].

The most popular solution to SLAM considers it a stochastic process in which the Extended Kalman Filter (EKF) is used to compute an estimation of a state vector $\mathrm{x}$ representing the sensor and environment feature locations, together with the covariance matrix $\mathbf{P}$ representing the error in the estimation. Most processes associated to the move-sense-update cycle of EKF SLAM are linear in the number of map features $n$ : vehicle prediction and inclusion of new features [9], [10]. The exception is the update of the covariance matrix $\mathbf{P}$ of the stochastic state vector that represents the vehicle and map states, which is $O\left(n^{2}\right)$. The EKF solution to SLAM has been used successfully in small-scale environments; however, the $O\left(n^{2}\right)$ computational complexity limits the use of EKF SLAM in large environments. This has been a subject of much interest in research. Postponement [11], the Compressed EKF Filter [10], the Sparse Weight Kalman filter [12], and Map Joining SLAM [13] are alternatives that work on local areas of the stochastic map and are essentially constant most of the time, although they require periodical $O\left(n^{2}\right)$ updates. Given a certain environment and sensor characteristics, an optimal local map size can be derived to minimize the total computational cost [14]. Recently, researchers have pointed out the approximate sparseness of the information matrix $\mathbf{Y}$, the inverse of the full covariance matrix $\mathbf{P}$. This suggests using the extended information filter, the dual of the extended Kalman filter, for SLAM updates. The Sparse Extended Information Filter (SEIF) algorithm [15] approximates the information matrix by a sparse form that allows $O(1)$ updates on the information vector. Nonetheless, data association becomes more difficult when the state and covariance matrix are not available, and the approximation can yield overconfident estimations of the state [16]. This overconfidence is overcome by the Exactly Sparse Extended Information Filter (ESEIF) [17] with a strategy that produces an exactly sparse information matrix with no introduction of inaccuracies through sparsification, but discarding odometry information.

The Thin-Junction Tree filter algorithm [18] works on the Gaussian graphical model represented by the information matrix, and achieves high scalability by working on an approximation, where weak links are broken. The Treemap algorithm [19] 
is a closely related technique: in cases where there are many overlapping features, an optional optimization uses a weak link breakage policy. Recently, the Exactly Sparse Delayed State filter [20] and Square Root Smoothing and Mapping (SAM) [21] provided the insight that the full SLAM problem, the complete vehicle trajectory plus the map, is sparse in information form (although ever increasing) allowing the use of sparse linear algebra techniques. The Tectonic SAM algorithm [22] provides a local mapping version to reduce the computational cost. However, the method remains a batch algorithm and covariance is not available to solve data association. The fast incremental Smoothing and Mapping (iSAM) algorithm [23] addresses the data association problem by recovering the exact covariance using QR-factorization on the information matrix. The authors report that iSAM is real time for the Victoria Park dataset (although EKF SLAM is also real time for this dataset).

A second important limitation of standard EKF SLAM is the effect that linearizations have in the precision and consistency of the final vehicle and feature estimates. Linearizations introduce errors in the estimation process that reduce precision and can render the result inconsistent, in the sense that the computed state covariance does not represent the real error in the estimation [24]-[26]. Among other things, this complicates data association, which is based on contrasting predicted feature locations with observations made by the sensor. Thus, important processes in SLAM like loop closing are complicated. All algorithms for EKF SLAM based on efficiently computing an approximation of the EKF solution will inevitably suffer from this consistency problem [18], [19]. The Unscented Kalman filter [27] avoids linearizations via a parameterization of means and covariances through selected points to which the nonlinear transformation is applied. Unscented SLAM has been shown to have improved consistency properties [28]. These solutions however ignore the computational complexity problem.

In this paper, we describe Divide and Conquer SLAM (D\&C SLAM), an EKF SLAM algorithm that overcomes these two fundamental limitations.

1) The computational cost per step is reduced from $O\left(n^{2}\right)$ to $O(n)$; the total cost of SLAM is reduced from $O\left(n^{3}\right)$ to $O\left(n^{2}\right)$.

2) The resulting vehicle and map estimates are more precise than with standard EKF SLAM and the computed state covariance more adequately represents the real error in the estimation.

Unlike many current large-scale EKF SLAM techniques, this algorithm computes a solution without relying on approximations or simplifications (other than linearizations) to reduce computational complexity. Also, estimates and covariances are available when needed by data association without any further computation. The Victoria Park dataset is used to test this algorithm. It produces better results than other state of the art SLAM methods [23], [29] for this dataset.

In a recent paper [30], the authors prove that convergence properties known to hold for linear EKF SLAM [31] hold for nonlinear EKF SLAM only when Jacobians (and thus linearizations) are computed around the ground truth solution, which is, in general, unfeasible. It is also proven that the use of Jacobians computed around the estimated value may result in overconfident estimates. In particular, when the robot orientation uncertainty is large, the extent of inconsistency is significant. Likewise, when the robot orientation uncertainty is small, the extent of inconsistency is insignificant. The authors point out that since the robot orientation error is the main source of inconsistency, algorithms that use local submapping, where the robot orientation error remains small, have the potential to improve consistency. Here, we confirm the consistency improvement offered by one of such local submapping algorithms.

This paper is organized as follows. In Section II, we briefly review the standard EKF SLAM algorithm and its computational properties. We also discuss other recent alternative algorithms, based on local mapping, with reduced computational cost. In Section III, we describe the D\&C SLAM algorithm and study its computational cost as well as its consistency properties in comparison with EKF SLAM and map joining SLAM. In Section IV, we compare the computational cost, precision and consistency of EKF SLAM and D\&C SLAM using simulated experiments. In Section V we analyze the computational cost of continuous data association in EKF SLAM, and describe the Randomized Joint Compatibility (RJC) algorithm for carrying out data association in D\&C SLAM also in linear time. In Section VI, we use the Victoria Park dataset to carry out an experimental comparison between EKF SLAM and D\&C SLAM. Finally, in Section VII, we draw the main conclusions of this paper.

\section{EKF SLAM ALGORITHM}

The EKF SLAM algorithm (see Algorithm 1) has been widely used for mapping and localization. Several authors have described the computational complexity of this algorithm [9], [10]. With the purpose of comparing EKF SLAM with the proposed D\&C SLAM algorithm, in this section, we briefly analyze the computational complexity of EKF SLAM.

\section{A. Computational Complexity of EKF SLAM}

For simplicity, assume that in the environment being mapped, features are distributed more or less uniformly. If the vehicle is equipped with a sensor of limited range and bearing, the amount of measurements obtained at any location will be more or less constant. Assume that at some step $k$, we have the following:

1) The map contains $n$ features.

2) The sensor provides $m$ measurements.

3) $r$ of these measurements correspond to reobserved features.

4) $s=m-r$, correspond to new features.

In an exploratory trajectory, $r$ and $s$ remain constant. Therefore, the size of the map is proportional to the number of steps that have been carried out.

1) Computational Cost Per Step: the computational complexity of carrying out the move-sense-update cycle of algorithm ekf_slam at step $k$ involves the following:

a) computing the predicted map $\hat{\mathbf{x}}_{k \mid k-1}, \mathbf{P}_{k \mid k-1}$, which requires obtaining the corresponding Jacobians $\mathbf{F}_{k}, \mathbf{G}_{k}$ also; 


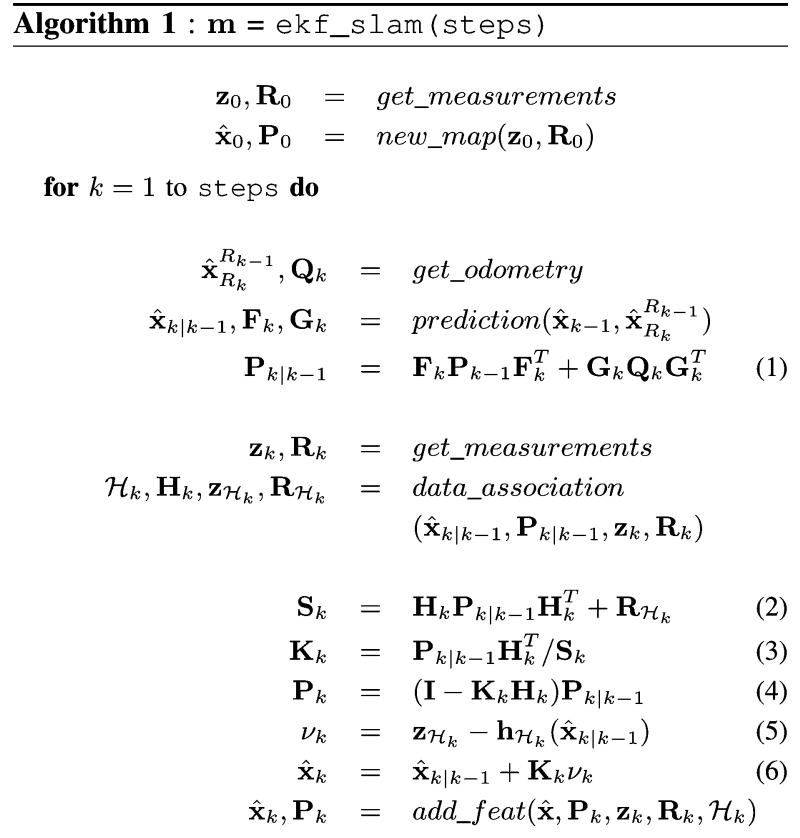

end for

return $\mathbf{m}=\left(\mathbf{x}_{k}, \mathbf{P}_{k}\right)$

b) solving data association (the complexity of data association is analyzed in Section V);

c) computing the updated map $\hat{\mathbf{x}}_{k}, \mathbf{P}_{k}$, which requires the computation of the corresponding Jacobian $\mathbf{H}_{k}$, the Kalman gain matrix $\mathbf{K}_{k}$, as well as the innovation $\nu_{k}$, and its covariance $\mathbf{S}_{k}$.

The fundamental fact regarding computational complexity in standard EKF SLAM is that, given a sensor of limited range and bearing, Jacobians matrices are sparse [9], [10], [21]: their computation is $O(1)$. But more importantly, since they take part in the computation of both the predicted and updated maps, the computational cost of (1)-(6) can also be reduced. Consider as an example the innovation covariance matrix $\mathbf{S}_{k}$ in (2). Without considering sparseness, the computation of this $r \times r$ matrix would require $r n^{2}+r^{2} n$ multiplications and $r n^{2}+r^{2} n+r^{2}$ sums, i.e., $O\left(n^{2}\right)$ operations. But given that matrix $\mathbf{H}_{k}$ is sparse, with an effective size of $r \times c$, the computation requires $r c n+r^{2} c$ multiplications and $r c n+r^{2} c+r^{2}$ sums, i.e., $O(n)$ operations (see Fig. 1, top). Similar analysis leads to the conclusion that the cost of computing both the predicted covariance $\mathbf{P}_{k \mid k-1}$ and the Kalman gain matrix $\mathbf{K}_{k}$ is $O(n)$, and that the greatest cost in an EKF SLAM update is the computation of the covariance matrix $\mathbf{P}_{k}$, which is $O\left(n^{2}\right)$. Thus, the computational cost per step of EKF SLAM is quadratic on the size of the map

$$
C_{\mathrm{EKF}, k}=O\left(n^{2}\right) \text {. }
$$

2) Total Computational Cost: Considering the earlier assumptions, during an exploratory trajectory, a constant number of $s$ new features are added to the map at each step. Thus, to map an environment of size $n, n / s$ steps are required, and the total cost of EKF SLAM will be

$$
C_{\mathrm{EKF}}=\sum_{k=1}^{n / s} O\left((k s)^{2}\right)=\sum_{k=1}^{n / s} O\left(k^{2}\right) .
$$
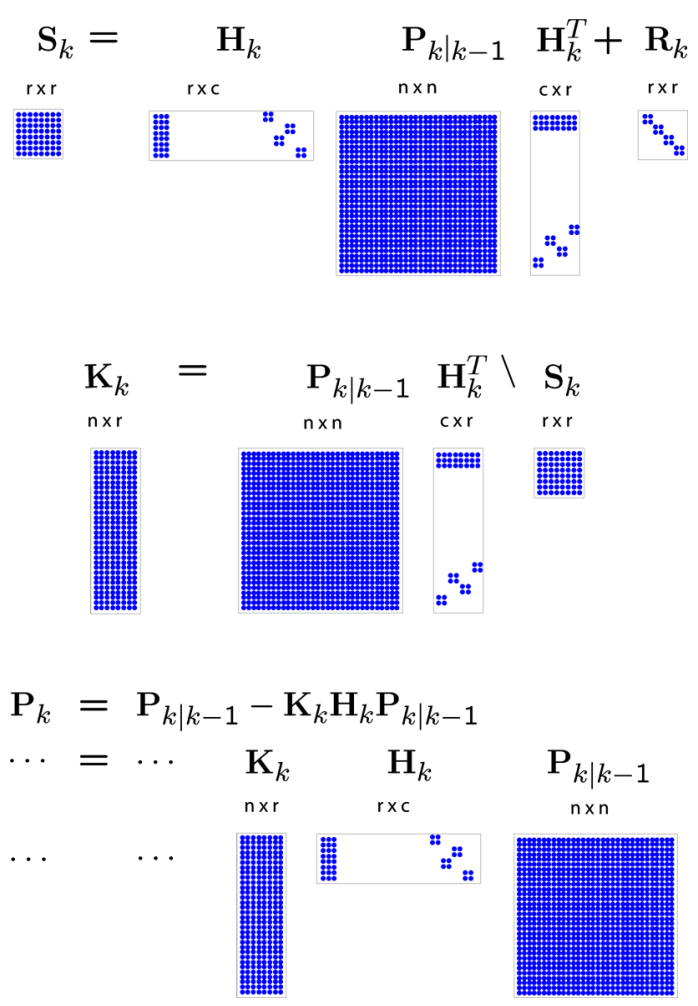

Fig. 1. (Top) Computation of the innovation covariance $\mathbf{S}_{k}$ matrix. Given that the effective size of the Jacobian matrix $\mathbf{H}_{k}$ is $r \times c$, the computation requires $O(n)$ operations $\left(r c n+r^{2} c\right.$ multiplications and $r c n+r^{2} c+r^{2}$ sums). In a similar way, (middle) the computations of the Kalman gain $\mathbf{K}_{k}$ matrix requires $O(n)$ operations and (bottom) the covariance matrix $\mathbf{P}_{k}$ requires $O\left(n^{2}\right)$ operations.

The square power summation is known to be

$$
\sum_{i=1}^{j} i^{2}=\frac{j(j+1)(2 j+1)}{6} .
$$

Thus, the total cost of EKF SLAM is cubic

$$
\begin{aligned}
C_{\mathrm{EKF}} & =O\left(\frac{(n / s)(n / s+1)(2 n / s+1)}{6}\right) \\
& =O\left(\frac{n^{3}}{s^{3}}\right) \\
& =O\left(n^{3}\right) .
\end{aligned}
$$

In general, the rate at which a map grows depends on the trajectory that the vehicle follows and the density of features in the environment. During exploration, the number of features in the current map will grow linearly, but when the vehicle returns retracing its steps, the map size will remain more or less constant. In these cases, the cost of updating the map is still $O\left(n^{2}\right)$ with the size of the map, but since $n$ does not increase, the total cost may be less than cubic.

\section{B. Local Mapping Algorithms}

Local mapping techniques have been proposed as computationally efficient alternatives to EKF SLAM. Instead of working on a full global map all the time as EKF SLAM does, a sequence 


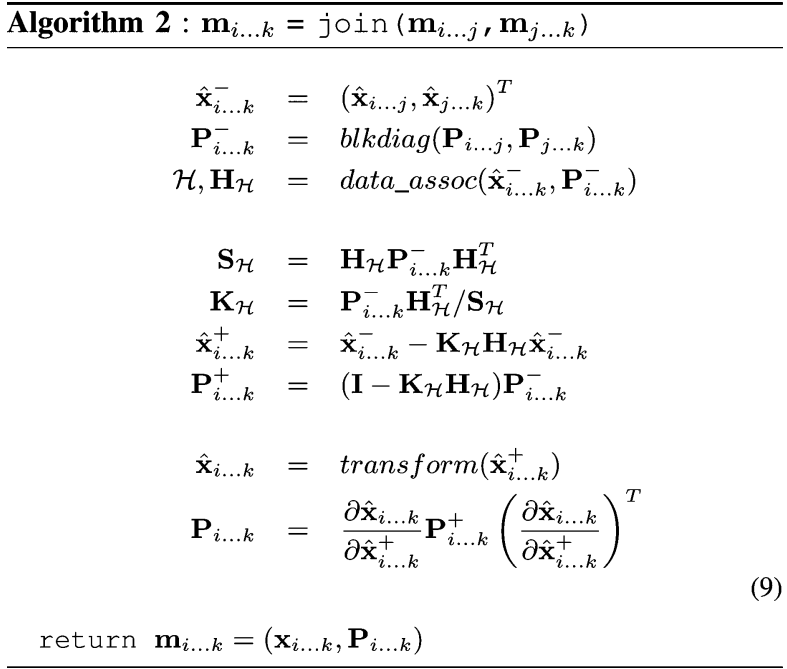

of local maps of limited size is produced. When the size of the current local map reaches some limit, the map is closed and stored, and a new local map is created. This allows us to maintain the computational cost constant most of the time while working on the current local map. Algorithms based on this idea include suboptimal or approximate solutions such as Decoupled Stochastic Mapping (DSM) [32], Constant Time SLAM (CTS) [33], the ATLAS framework [34], and Hierarchical SLAM [35]. These algorithms sacrifice precision in the resulting estimation of the map in order to maintain the computational cost linear in the size of the map at worst.

There are alternative solutions that do not carry out approximations, such as Map Joining SLAM [13] and the Constrained Local Submap Filter [36]. Given that we are interested in algorithms that do not sacrifice precision in order to limit computational cost, we concentrate on these two. Map Joining SLAM (and similarly, the constrained local submap filter) is an EKFbased algorithm in which a sequence of independent local maps of a limited size $p$ is produced using the standard EKF SLAM algorithm. Map independence is guaranteed by construction: once the current local map is closed, a new local map is initialized with zero covariance using the current vehicle pose as the base reference for the new map. These local maps are later fused using a map joining procedure to produce a single final stochastic map. Algorithm 2 details the map joining procedure. For two consecutive local maps $\mathbf{m}_{i, \ldots, j}$ and $\mathbf{m}_{j, \ldots, k}$, computed in steps $i, \ldots, j$ and $j, \ldots, k$, respectively, map joining computes the resulting map $\mathbf{m}_{i, \ldots, k}$ for all steps $i, \ldots, k$ in the following way:

1) Both maps are simply stacked together, with the features of each in each local base reference. Due to map independence, the cross-covariance between both maps is zero.

2) Data association is carried out to find common features between both maps.

3) Using a modified version of the EKF update equations the map is optimized by fusing common features.

4) All features are transformed to the base reference of the first map.

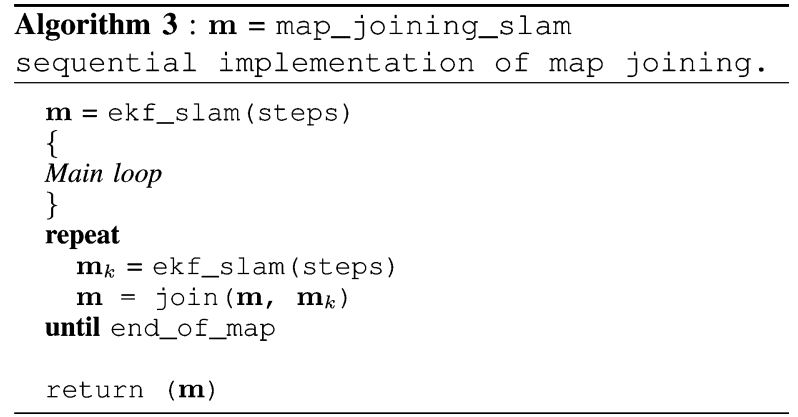

A more detailed explanation of this procedure and its notation can be found in the Appendix. It is worth noting that map joining SLAM never revisits a prior local map. Instead, a new local map is created, which will be joined later with the previous map to obtain a global map that includes all available information.

An analysis similar to that of Fig 1 shows that for local maps of limited size, the computational cost of map joining SLAM is $O\left(n^{2}\right)$ on the size of the resulting global map, again being the most expensive operation the update of the covariance matrix $\mathbf{P}$. However, map joining takes place only when a given local map has reached its limit size. In all other steps, only a local map is being updated, with a computational cost of $O(1)$.

Algorithm 3 carries out Map Joining SLAM: ekf_slam is used to compute a local map $\mathbf{m}_{k}$ of a given limit size (or for a given limited number of steps). This local map is joined to a global map $\mathbf{m}$ by means of the join function in a sequential fashion. The process continues until the environment is completely covered.

\section{DIVIDE AND CONQUER AlgorithM}

Instead of joining each new local map to a global map sequentially, as map joining SLAM does, the algorithm proposed in this paper, Divide and Conquer SLAM, carries out map joining in a hierarchical fashion, as depicted in Fig. 2. The lower nodes of the hierarchy represent the sequence of $l$ local maps of minimal size $p$, computed with standard EKF SLAM. These maps are joined pairwise to compute $l / 2$ local maps of double their size $(2 p)$, which will in turn be joined pairwise into $l / 4$ local maps of size $4 p$, until finally two local maps of size $n / 2$ will be joined into one full map of size $n$, the final map size. D\&C is implemented using Algorithm 4, which uses a stack to save intermediate maps. Whenever two maps of around the same size are at the top of the stack, they are replaced in the stack by their join. This allows a sequential execution of D\&C SLAM.

\section{A. Total Computational Complexity of D\&C SLAM}

In D\&C SLAM, the process of building a map of size $n$ produces $l=n / p$ maps of size $p$ (not considering overlap), at cost $O\left(p^{3}\right)$ each [see (8)], which are joined into $l / 2$ maps of size $2 p$, at cost $O\left((2 p)^{2}\right)$ each. These in turn are joined into $l / 4$ maps of size $4 p$, at cost $O\left((4 p)^{2}\right)$ each. This process continues until two local maps of size $n / 2$ are joined into one local map 


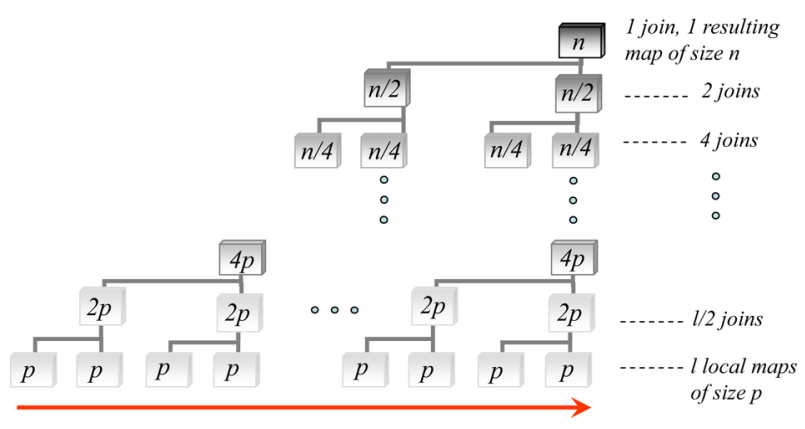

Fig. 2. Hierarchy of maps that are created and joined in D\&C SLAM. The lower level is the sequence of $l$ local maps of size $p$ computed with standard EKF SLAM, as the arrow suggests. The intermediate levels represent intermediate joins during the process. The top level represents the final map of size $n$ resulting from the join of two local maps of size $n / 2$.

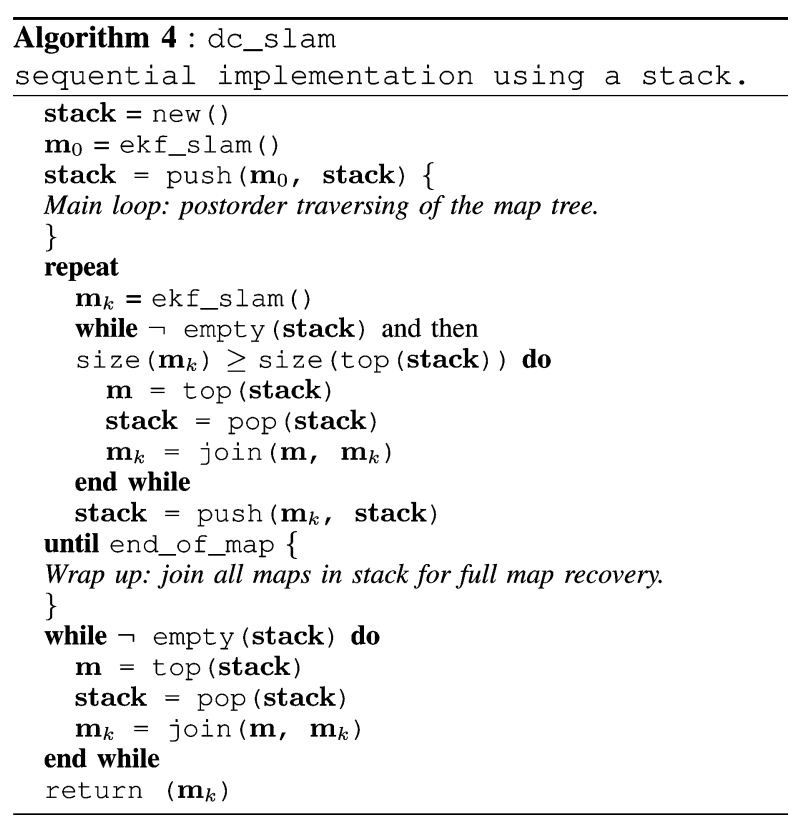

of size $n$, at a cost of $O\left(n^{2}\right)$. Map joining SLAM and D\&C SLAM carry out the same number of map joining operations. The fundamental difference is that in D\&C SLAM, the size of the maps involved in map joining increases at a slower rate than in Map Joining SLAM. As shown next, this allows the total cost to remain quadratic with $n$.

The total computational complexity of D\&C SLAM is

$$
\begin{aligned}
C_{\mathrm{DC}} & =O\left(p^{3} l+\sum_{i=1}^{\log _{2} l} \frac{l}{2^{i}}\left(2^{i} p\right)^{2}\right) \\
& =O\left(p^{3} n / p+\sum_{i=1}^{\log _{2} n / p} \frac{n / p}{2^{i}}\left(2^{i} p\right)^{2}\right) \\
& =O\left(p^{2} n+\sum_{i=1}^{\log _{2} n / p} p \frac{n}{2^{i}}\left(2^{i}\right)^{2}\right) \\
& =O\left(p^{2} n+p n \sum_{i=1}^{\log _{2} n / p} 2^{i}\right) .
\end{aligned}
$$

Note that the sum represents all costs associated to map joining. This corresponds to the sum of a geometric progression of the type

$$
\sum_{i=1}^{k} r^{i}=\frac{r-r^{k+1}}{1-r} .
$$

Thus, in this case

$$
\begin{aligned}
C_{\mathrm{DC}} & =O\left(p^{2} n+p n \frac{2^{\log _{2} n / p+1}-2}{2-1}\right) \\
& =O\left(p^{2} n+p n\left(\frac{2 n}{p-2}\right)\right) \\
& =O\left(p^{2} n+2 n^{2}-2 p n\right) \\
& =O\left(n^{2}\right) .
\end{aligned}
$$

This means that D\&C SLAM performs SLAM with a total cost quadratic with the size of the environment, as compared with the cubic cost of standard EKF SLAM. This corresponds to the normal exploration operation. In the worst-case scenario, when the overlap between the maps to be joined is full (i.e., if the robot traverses the whole map for a second time), the cost of map joining will be cubic, the same as EKF SLAM.

\section{B. Computational Complexity of D\&C SLAM Per Step}

In D\&C SLAM, in steps that are a power of 2 , when $k=$ $2^{t}, t$ joins will take place, at a cost $O\left(2^{2}\right), O\left(4^{2}\right), \ldots, O\left(k^{2}\right)$, respectively. An analysis similar to that of (10) shows that the total cost of such steps is $O\left(k^{2}\right)$, which is of the same order as a standard EKF SLAM step. However, in D\&C SLAM, the map to be generated at step $k$ will not be required for joining until step $2 k$. This allows us to amortize the cost $O\left(k^{2}\right)$ at this step by dividing it up between steps $k+1$ to $2 k$ in equal $O(k)$ computations for each step. In this way, amortized D\&C SLAM becomes a linear-time SLAM algorithm.

An amortized version of D\&C SLAM can be implemented using two concurrent threads: one high-priority thread executes ekf_slam (Algorithm 1) to update the current local map, and the other low-priority thread executes dc_slam (Algorithm 4). In this way, all otherwise idle time in the processor will be used for the more costly map joining operations, but high priority is given to local mapping, allowing for real-time execution of D\&C SLAM.

As we will see in the Monte Carlo simulations and the experiments, the amortized cost of D\&C SLAM is always lower than that of EKF SLAM. If at any moment during the map building process, the full map is required for another task, it can be computed in a single $O\left(n^{2}\right)$ step.

\section{SIMULATED EXPERIMENTS}

We use four simulated scenarios (see Fig. 3) to illustrate the properties of the algorithms discussed in this paper. In an environment that consists of equally spaced point features, a 

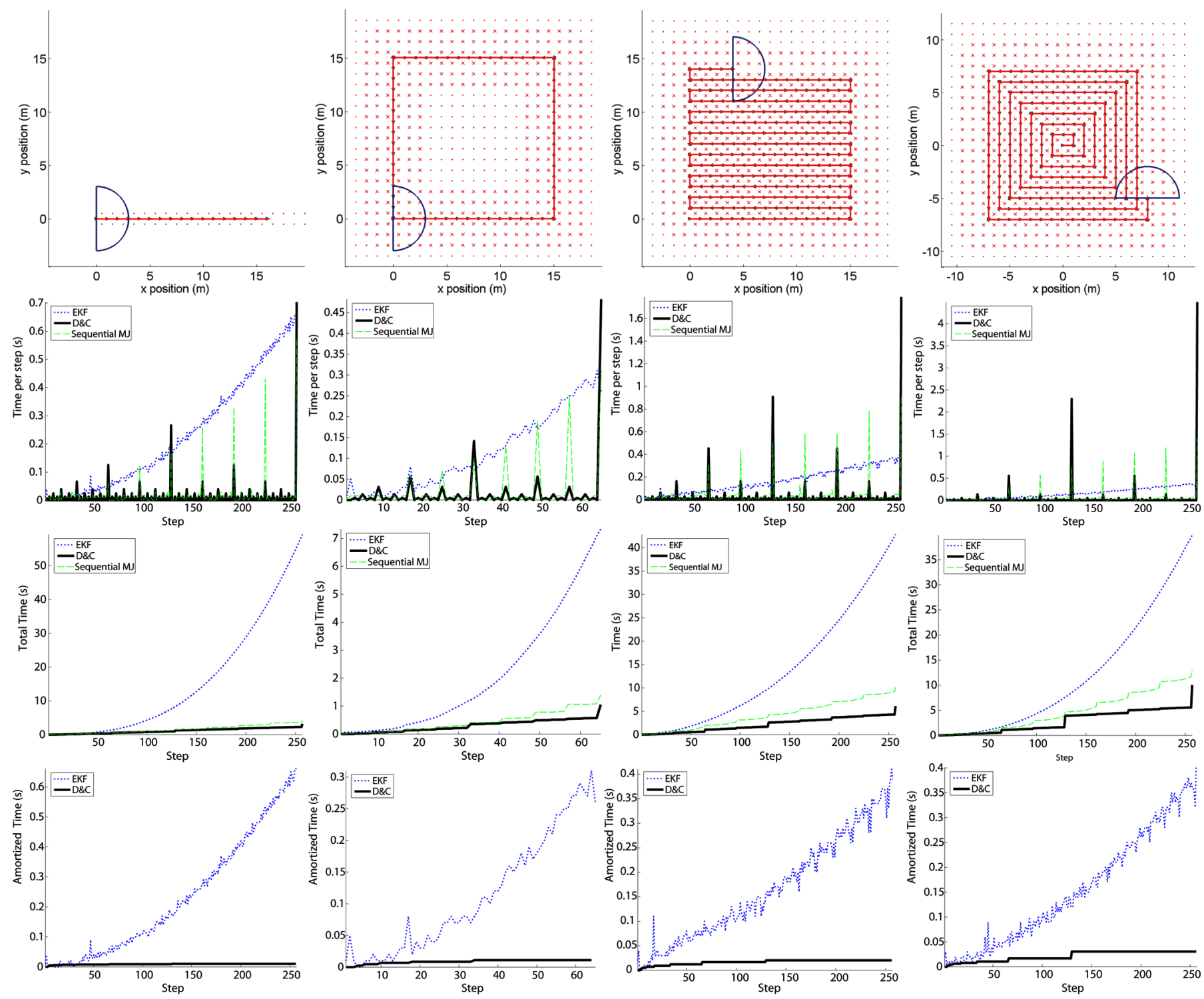

Fig. 3. Four simulated experiments for comparing the EKF, Sequential Map Joining and D\&C SLAM algorithms. (First column) Straighforward trajectory; (second column) loop closing; (third column) lawn mowing; (fourth column) outward spiral. (Top row) Ground truth environment and trajectory. (Second row) Execution time per step of EKF versus Map Joining versus D\&C SLAM. (Third row) Total execution time. (Bottom row) Execution time per step of EKF SLAM versus amortized execution time per step of D\&C SLAM.

vehicle equipped with a range and bearing sensor carries out four different trajectories: a straight line, a square loop, lawn mowing, and outward spiral (first, second, third, and fourth columns, respectively). The simulated experiments were carried out with known data association for the evaluated algorithms, in order to discard mismatching effects in the resulting performance of the estimators. The first row shows the environment and each of the trajectories. The second and third rows show the execution time per step and the total execution time, respectively. It can be seen that the total cost of D\&C SLAM quickly separates from the total cost of EKF SLAM, and also from the Map Joining SLAM. The reason is that the computational cost per step of D\&C SLAM is lower than that of EKF SLAM most of the time. EKF SLAM works with a map of nondecreasing size, while D\&C SLAM works on local maps of small size most of the time. Map joining SLAM is computationally equivalent to D\&C SLAM when working on local maps. In steps that are a power of two, the computational cost of D\&C is higher. In those steps, one or more map joining operations take place (in steps $2^{t}, t$ map joining operations take place). The accompanying AVI videos dcslam_xvid_loop.avi, dcslam_xvid_lawn.avi and dcslam_xvid_spiral.avi (available at http://ieeexplore.ieee.org) show the execution of both EKF SLAM and D\&C SLAM for the same sample data. The frames have been time stamped so that the actual running times of the algorithms in our Matlab implementation are shown.

Fig. 3 (bottom row) shows the amortized cost per step for the four simulated experiments. We can see that the amortized cost of D\&C SLAM is always lower than that of EKF SLAM. 

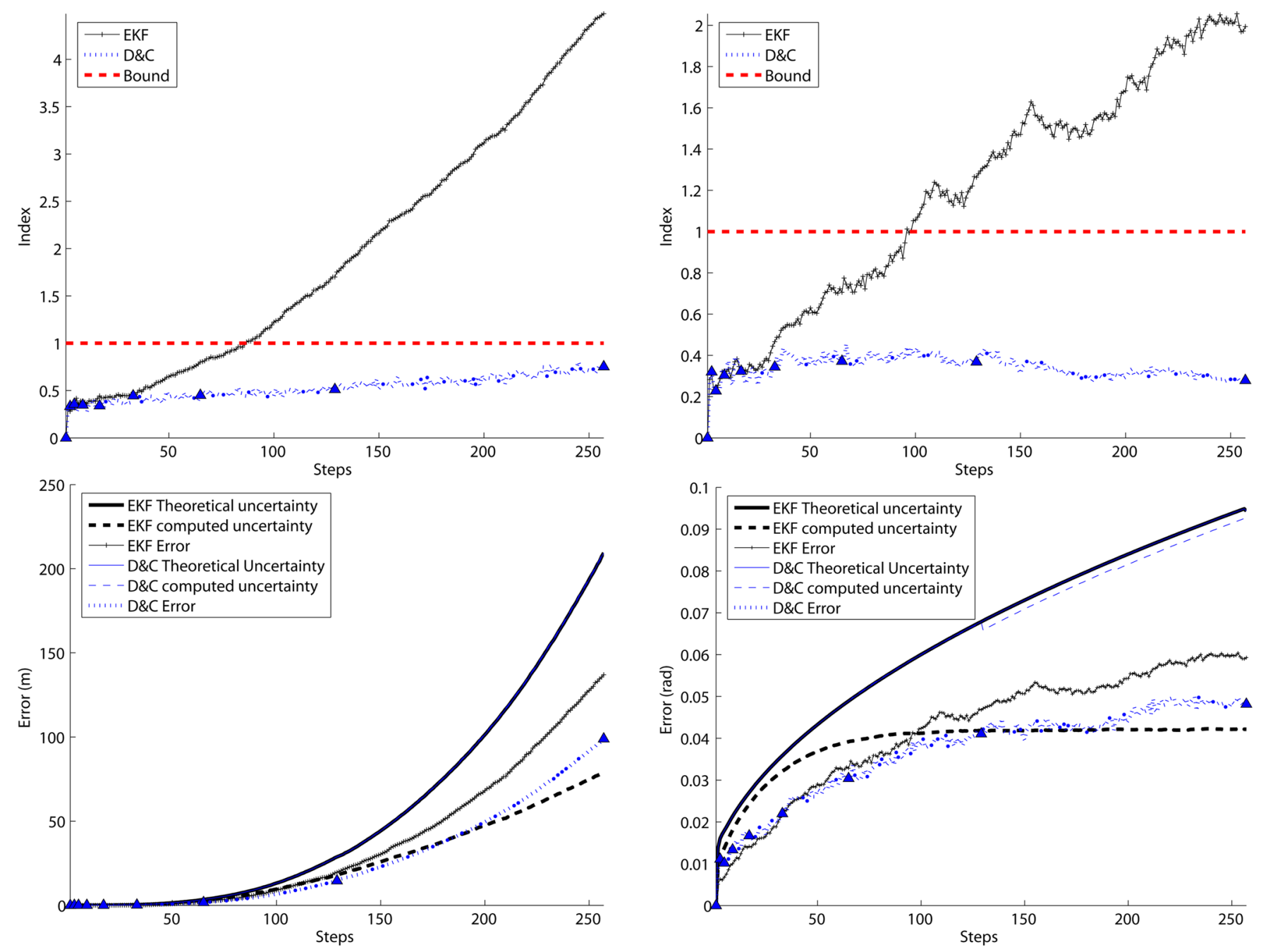

Fig. 4. (Top) Mean consistency index CI of (13) and (bottom) root-mean-squared error for the (left) robot x-y position and (right) orientation for standard EKF and D\&C SLAM. The root-mean-square error is always smaller for D\&C SLAM; also, the computation of the variance of the error is more precise, and thus, the estimation always remains consistent. The EKF and D\&C theoretical uncertainties coincide over all steps. Also, D\&C computed uncertainty superimposes the latter two.

\section{A. Consistency and Precision in Divide and Conquer SLAM}

Apart from computational complexity, another important aspect of the solution computed by the EKF has gained attention recently: map consistency and precision. When the ground truth solution $\mathrm{x}$ for the state variables is available, a statistical test for filter consistency can be carried out on the estimation $(\hat{\mathbf{x}}, \mathbf{P})$, using the Normalized Estimation Error Squared (NEES), defined as

$$
D^{2}=(\mathbf{x}-\hat{\mathbf{x}})^{T} \mathbf{P}^{-1}(\mathbf{x}-\hat{\mathbf{x}}) .
$$

Consistency is checked using a chi-square test:

$$
D^{2} \leq \chi_{r, 1-\alpha}^{2}
$$

where $r=\operatorname{dim}(\mathbf{x})$ and $\alpha$ is the desired significance level (we consider the usual $\alpha=0.05$ ). If we define the consistency index of a given estimation $(\hat{\mathbf{x}}, \mathbf{P})$ with respect to its true value $\mathbf{x}$ as

$$
\mathrm{CI}=\frac{D^{2}}{\chi_{r, 1-\alpha}^{2}}
$$

when $\mathrm{CI}<1$, the estimation is consistent with ground truth, and when CI $>1$, the estimation is inconsistent (optimistic) with respect to ground truth. Thus, CI measures how precise the computed covariance is with respect to the real error, while precision can be simply computed as the root of the squared difference with ground truth.

We tested consistency of both standard EKF and D\&C SLAM algorithms by carrying out 100 Monte Carlo runs on the simulated experiments. Simulation allows us to have ground truth available. Additionally, Monte Carlo runs allow to obtain statistically significant evidence about the consistency of the algorithms being compared.

Fig. 4 (top) shows the evolution of the mean consistency index of the vehicle position (left) and orientation (right) during all steps of the straight forward trajectory simulation. We can see that the D\&C estimate on vehicle location is always more consistent than the standard EKF estimate; in less than 100 steps, EKF falls out of consistency while D\&C remains consistent. Fig. 4 (bottom) shows the evolution of the root-mean-square error on the vehicle position and orientation. The $2 \sigma$ bounds 
This article has been accepted for publication in a future issue of this journal, but has not been fully edited. Content may change prior to final publication.
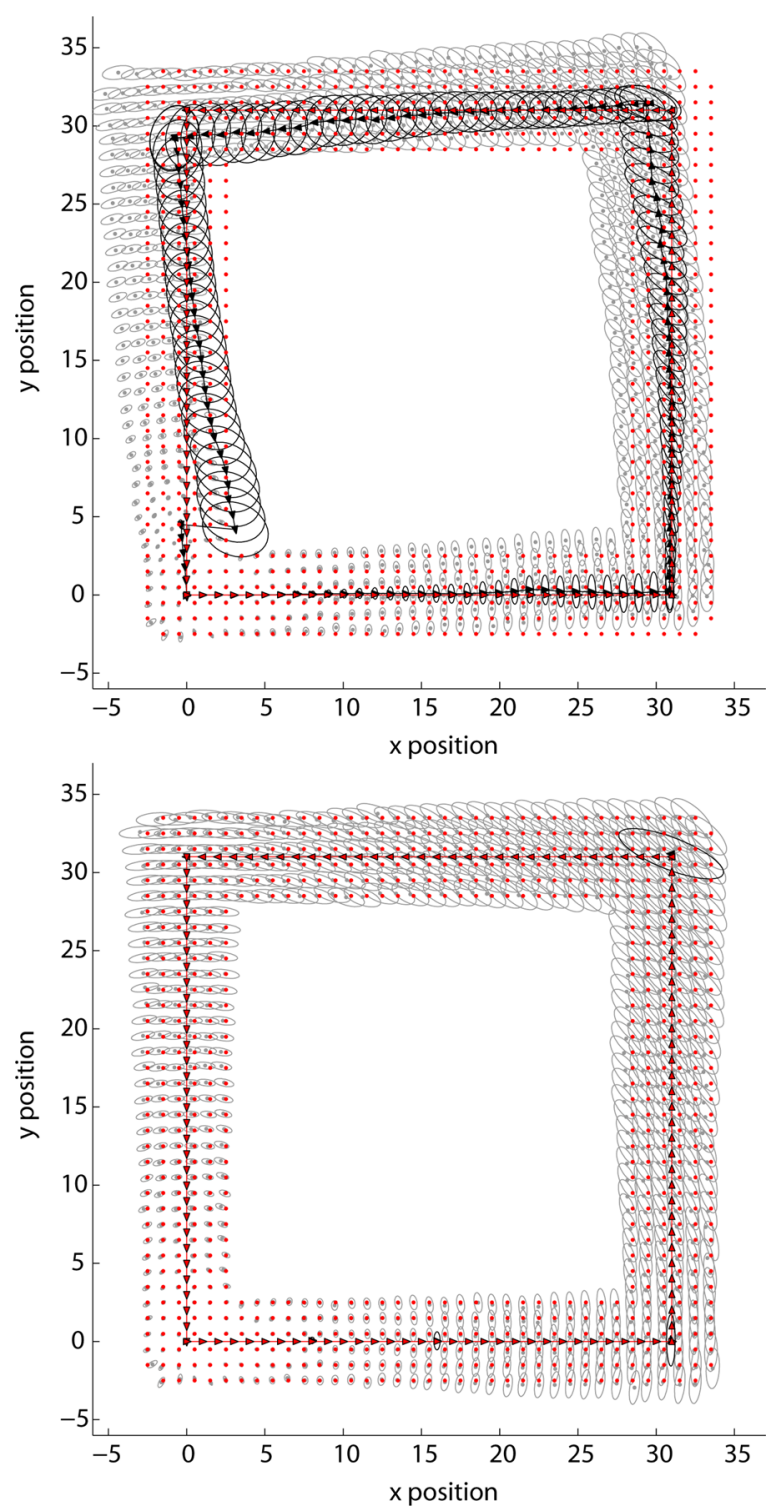

Fig. 5. (Top) Typical result when running EKF SLAM and (bottom) D\&C SLAM on the same data. The vehicle and map features tend to accumulate more error during exploration with EKF SLAM. Even if data association is known, the final result after closing a loop is less precise. The absolute vehicle location estimates are shown when available from each algorithm. Ground truth environment and trajectory are shown in red.

for the theoretical uncertainty are computed by running the simulated experiment without measurement and robot noise, so that linearizations take place in the true state values, and thus, introduce no errors. The computed uncertainty of both standard EKF and D\&C SLAM are also drawn. We can see how the RMS error increases more slowly in the case of D\&C SLAM. We can also see the fast rate at which the uncertainty computed by standard EKF SLAM falls below its theoretical value.

Monte carlo runs show that Divide and Conquer SLAM is less subject to linearization errors than EKF SLAM. Fig. 5 shows a typical situation: the two algorithms run on exactly the same data of a loop closure (the accompanying video dcslam_xvid_loop.avi shows the execution of the two algorithms for the same data). Because of less accumulated error, and thus, better linearizations, the final result is much more precise for D\&C SLAM (data association is known and used in both algorithms).

\section{DATA ASSOCIATION FOR DIVIDE AND CONQUER SLAM}

\section{A. Data Association for Standard EKF SLAM}

The data association problem in continuous EKF SLAM consists in producing a hypothesis $\mathcal{H}=\left[j_{1}, j_{2}, \ldots, j_{i} \ldots, j_{m}\right]$, where correspondences are established between each of the $i=1, \ldots, m$ sensor measurements and one (or none) of the $j=1, \ldots, n$ map features. The space of measurement-feature correspondences can be represented by an interpretation tree of $m$ levels [37]. Each node of the tree at level $i$ has $n+1$ branches, corresponding to the $n$ alternative feature pairings for measurement $i$, and an extra node (star-branch) to account for the possibility of the measurement being spurious or a new feature. The size of this correspondence space, (i.e., the number of alternative hypotheses) in which data association must be solved is exponential with the number of measurements: $(n+1)^{m}$.

Fortunately, the availability of a stochastic model for both the map and the measurements allows us to check each measurement-feature correspondence for individual compatibility by predicting the location of the map features relative to the sensor reference and determine compatibility using a hypothesis test on the innovation and covariance of each possible pairing.

The discrepancy between the observation $i$ and the predicted observation of map feature $j$ is measured using the innovation term of (5) and its covariance (2). The measurement can be considered corresponding to the feature if the Mahalanobis distance $D_{k, i j}^{2}$ satisfies [38]

$$
D_{k, i j}^{2}=\nu_{k, i j}^{T} \mathbf{S}_{k, i j}^{-1} \nu_{k, i j}<\chi_{d, 1-\alpha}^{2}
$$

where $d=\operatorname{dim}\left(\mathbf{h}_{k, j}\right)$ and $1-\alpha$ is the desired confidence level, usually $95 \%$.

In standard EKF SLAM, and for a sensor of limited range and bearing, the number of measurements $m$ is constant, and thus, individual compatibility is $O(n m)=O(n)$, linear on the size of the map. This cost can be easily reduced to $O(m)$, a constant, by a simple tessellation or grid of the map computed during map building, which allows us to determine candidates for a measurement in constant time simply by checking the grid element and nearby grid elements in which its predicted location falls. In 2-D problems, the cost of computing and updating the tessellation would be constant per step (a constant amount of new features are included in the map per step), while the space required would be proportional to the total area covered by the map and the resolution of the tessellation.

In cases where clutter or vehicle error are high, there may be more than one possible correspondence for each measurement. More elaborate algorithms are required to disambiguate in these cases. Nevertheless, the overlap between the measurements and the map is the size of the sensor range plus the vehicle uncertainty, and thus, more or less constant. In local mapping, after individual compatibility is sorted out, we use the Joint Compatibility Branch and Bound (JCBB) algorithm [39] (see Algorithm 5) 


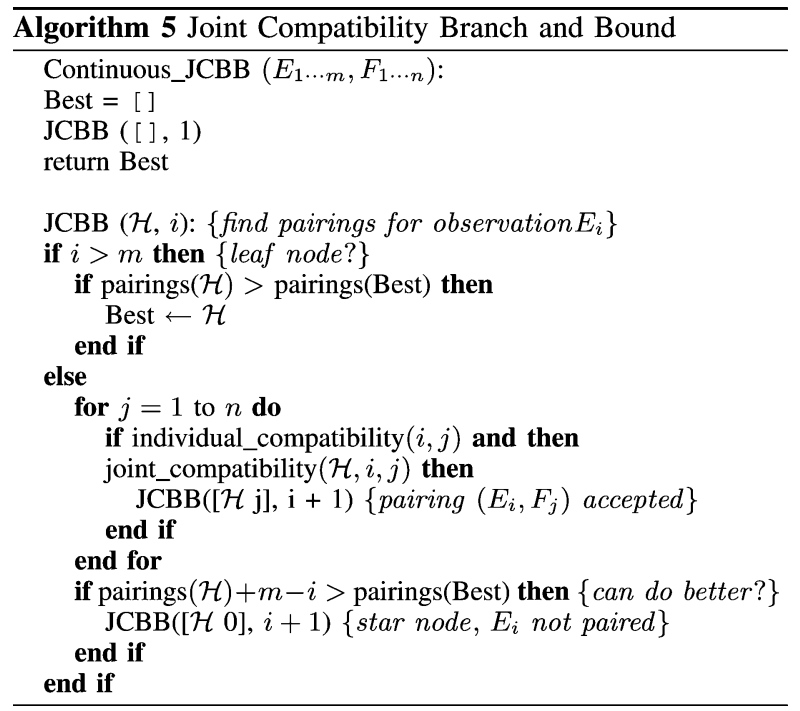

to disambiguate between the possible associations for the $m$ measurements. It has been shown that algorithms such as JCBB, based on a probabilistic model (feature estimates and covariances), can greatly increase the robustness of data association, even when other measurement properties are available. For instance, in the case of monocular SLAM, the combined use of texture-based matching and stochastic compatibility tests has proved critical to reject outliers, especially from repetitive patterns and dynamic objects [40]. JCBB performs branch and bound search on the interpretation tree looking for jointly compatible correspondences, but only in the overlap determined by individual compatibility. Given that this is a region of the map of constant size, each measurement will have a more or less constant number of feature candidates, say $c$, and thus, the solution space is constant: $(c+1)^{m}$. In this way, JCBB will execute in constant time.

\section{B. Data Association for Divide and Conquer SLAM}

Data association in D\&C SLAM is a very particular problem because it involves finding correspondences between two local maps of similar size whenever joining is to take place. For instance, before obtaining a final map of size $n$, the data association problem has to be solved between two maps of size $n / 2$, and so, computing individual compatibility would be $O\left(n^{2}\right)$ instead of $O(n)$. Fortunately, as in the case of individual compatibility for standard EKF SLAM, finding potential matches for one feature in another map can be done in constant time using a simple tessellation or grid in the map where the search is done. Consider the example in Fig. 6. The red trajectory and features correspond to the first local map built, and the blue trajectory and features correspond to the second local map. Individual compatibility may be done in a way similar to standard EKF SLAM: we predict the location of features in the first (red) map relative to the base reference of the second (blue) map, and check for possible correspondences with blue features. If the blue map is tessellated, we can find potential matches for a red feature in constant time, and for the whole red map in

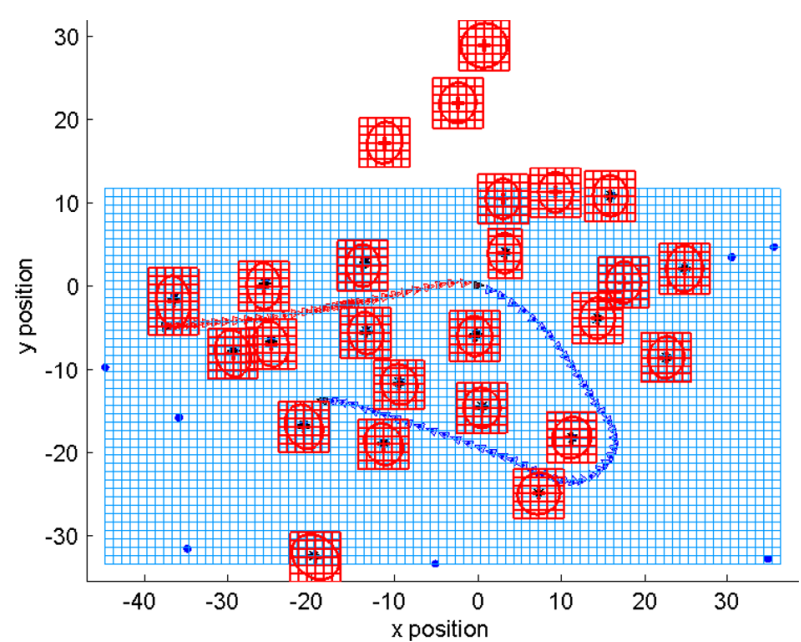

Fig. 6. Tessellation to compute individual compatibility between two local maps of similar size. The second (blue) map is tessellated using a grid. Red ellipses represent the uncertainties of the predicted features of the first local map with respect to the base reference of the second. The ellipses are approximated by windows, and in this way, possible candidates (asterisks) for each red feature can be found in constant time. The robot trajectory is also shown for each local map with the corresponding color.

linear time. The cost of computing and updating the tessellation is constant per step, while the space required is proportional to the total area covered by each map. Alternative solutions, such as 2-D priority kd-trees [41], can be used to make the storage space required to be $O(n \log n)$ on the number of map features, instead of being dependent on the covered area as the tessellation is. There is, however, a higher cost involved in finding a potential match per feature, $O(\log n)$, and an update cost of $O(\log n)$ per new feature.

A second issue of importance is the size of the region of overlap between two local maps. While in standard EKF SLAM, this region is constant, and thus, data association algorithms like JCBB will execute in constant time, in D\&C SLAM, the size of the overlap is not always constant. It basically depends on the environment and type of trajectory. Consider the simulated examples of Fig. 7, where two $n / 2$ maps are shown. In the first case, the square loop, the region of overlap between two maps, will be of constant size, basically dependent on the sensor range. In the case of the lawn mowers trajectory, the overlap will be proportional to the length of the trajectory before the vehicle turns back, basically the square root of $n$. In the third case, the outward spiral, the region of overlap between the inner map and the encircling map is proportional to the square root of $n$ as well. In some cases, like traversing a loop for a second time, the size of the overlap is the entire second local map.

In order to limit the computational cost of data association between local maps in D\&C SLAM, we use a randomized joint compatibility (RJC) algorithm. Our RJC approach (see Algorithm 6) is a variant of the linear RS algorithm [42] used for global localization. Consider two consecutive maps $\mathbf{m}_{1}$ and $\mathbf{m}_{2}$ of size $n_{1}$ and $n_{2}$, respectively, to be joined. First, the overlap between the two maps is identified using individual compatibility. Second, instead of performing branch and bound interpretation 

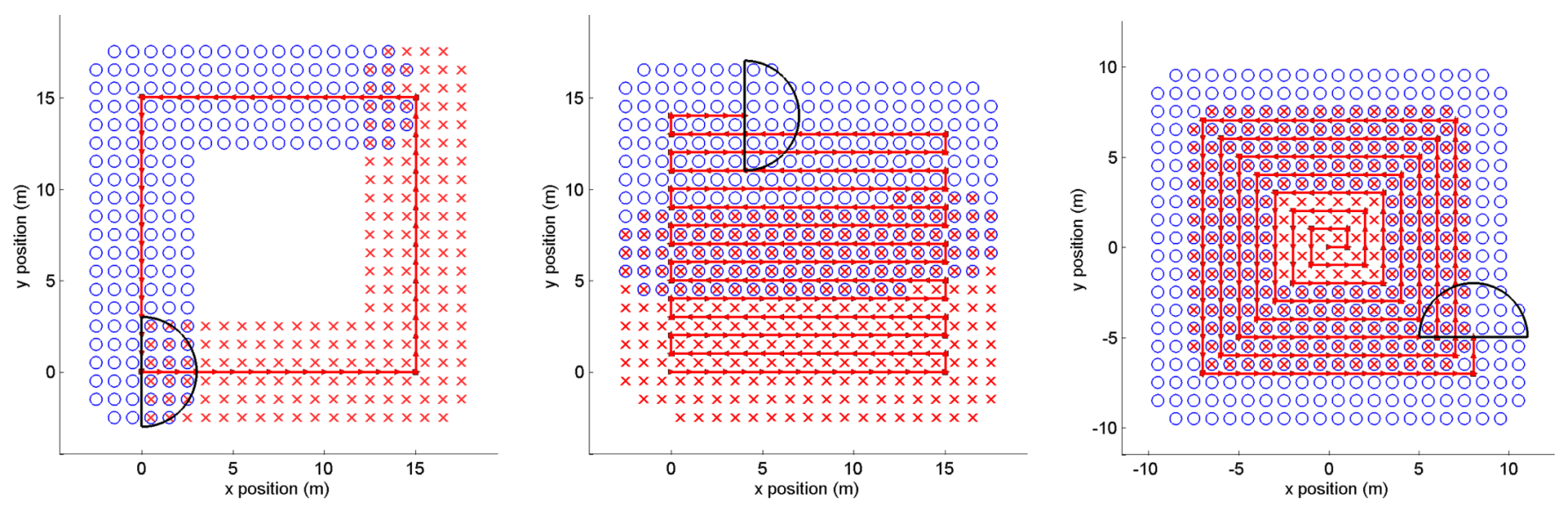

Fig. 7. Size of the overlap between two final local maps, i.e., common features in both maps. Crosses correspond to the first map and circles to the second map. (Left) Square loop trajectory (middle) lawn mowers trajectory, and (right) outward spiral.

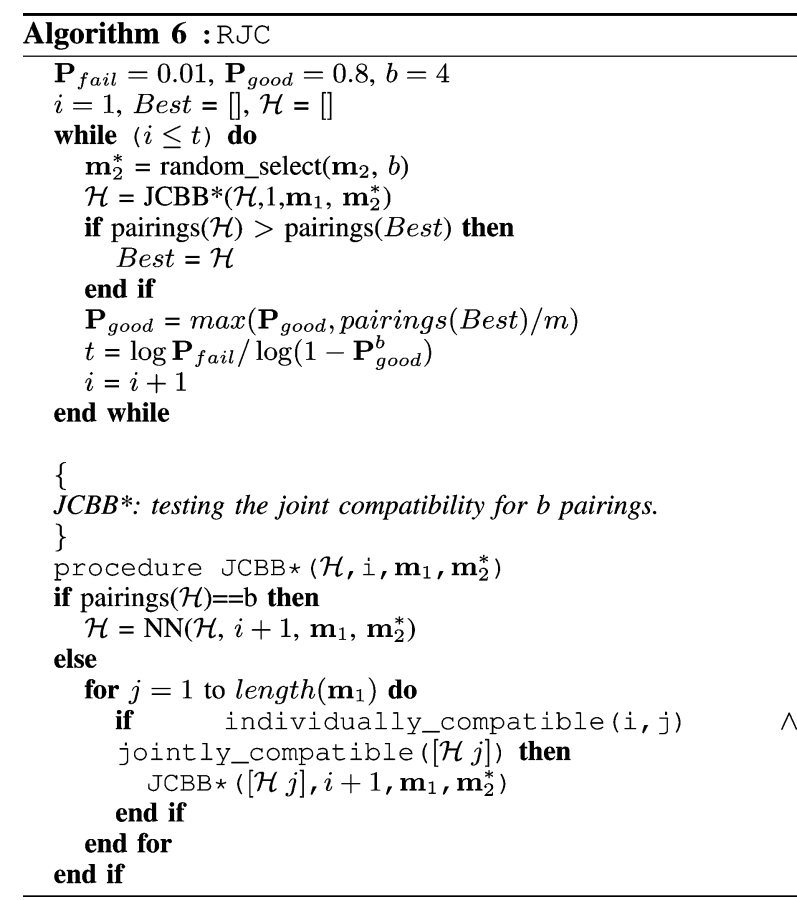

tree search in the whole overlap as in JCBB, we randomly select $b$ features in the overlapped area of the second map and use JCBB*. This algorithm is a version of JCBB, where all $b$ features are expected to be found in the second map (no star branch). This produces a hypothesis $\mathcal{H}$ of $b$ jointly compatible features in the first map. Associations for the remaining features in the overlap are obtained using the simple nearest neighbor rule given hypothesis $\mathcal{H}$, i.e., finding pairings that are compatible with the first $b$ features. In the spirit of adaptive random sample consensus (RANSAC) [43], we repeat this process $t$ times, so that the probability of missing a correct association is limited to $P_{\text {fail }}$.

The RJC algorithm successfully detects the overlap between two local maps in either continuous data association or loop closing. The only requirement is that the stochastic maps remain consistent, a condition that is enforced by the $\mathrm{D} \& \mathrm{C}$ algorithm.

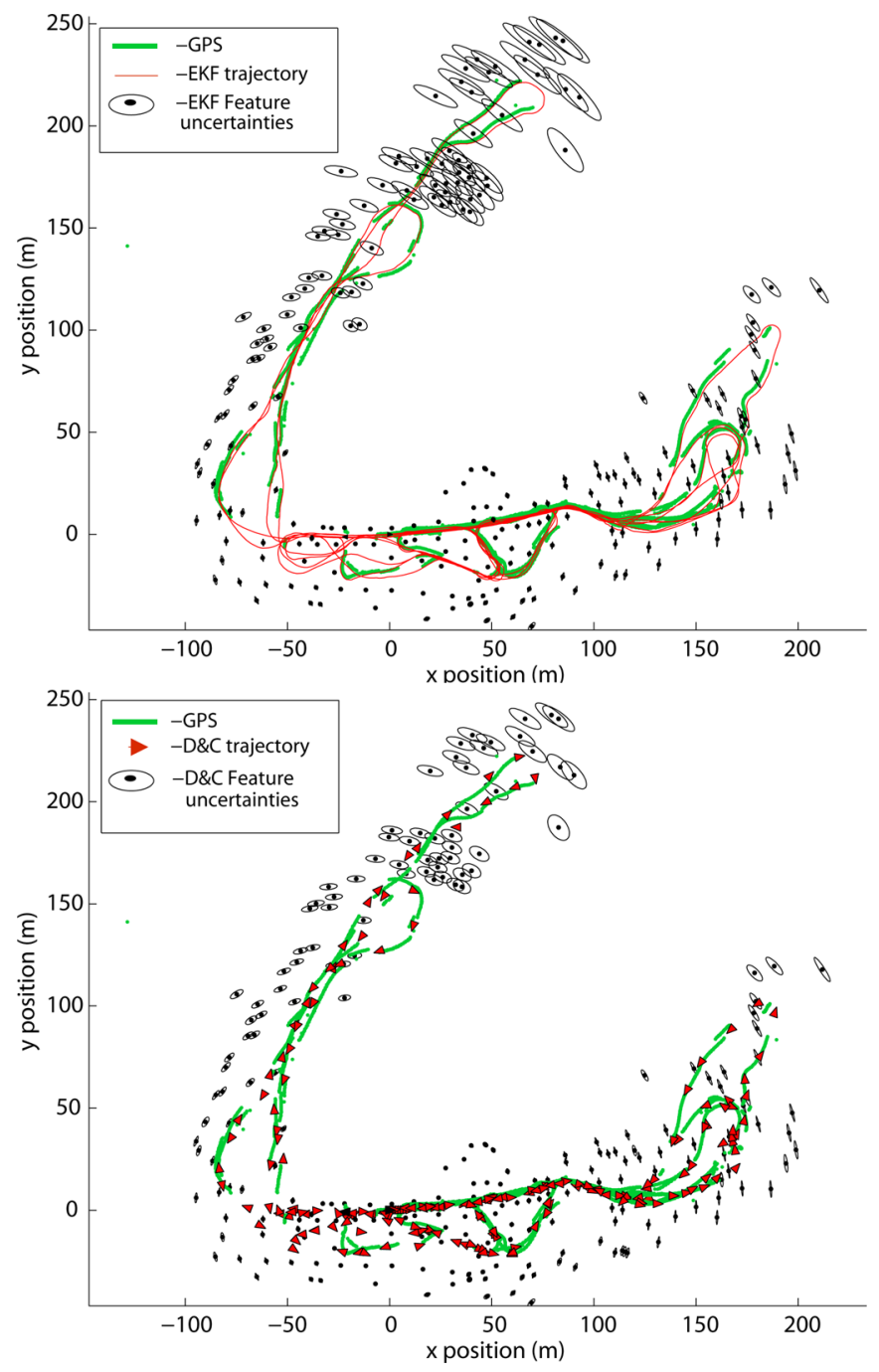

Fig. 8. (Top) Map for Victoria Park dataset according to the EKF SLAM and (bottom) D\&C SLAM. The results are essentially equivalent; there are some minor differences due to missed associations in the case of EKF. The estimated position along the whole trajectory is shown as a red line for EKF SLAM, and the vehicle locations are drawn as red triangles when available in D\&C SLAM. Green points are GPS readings in both cases and are not used in either case. 


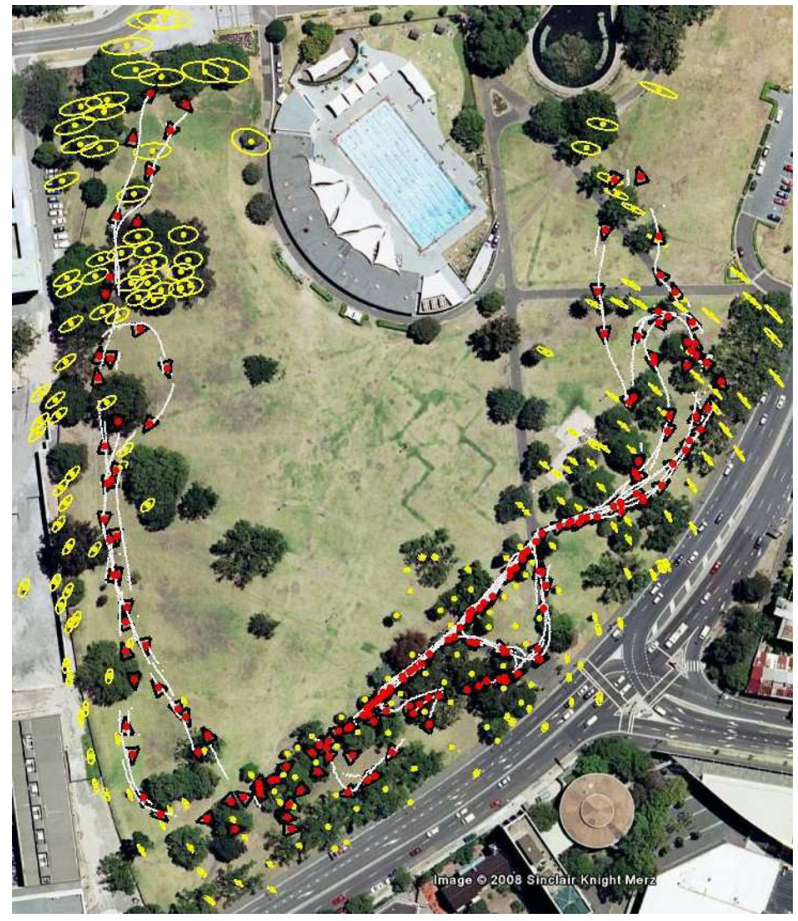

Fig. 9. The results were projected on Google Earth in order to compare the precision obtained.

Since JCBB* is executed using a fixed number of features, its cost remains constant. Finding the nearest neighbor for each remaining feature among the ones that are individually compatible with it, a constant number, will be constant. The cost of each try is thus $O(n)$. The number of tries depends on $b$, the number of features randomly selected, on the probability that a selected feature in the overlap can be actually found in the first map $P_{\text {good }}$, and on the acceptable probability of failure in this probabilistic algorithm, $P_{\text {fail }}$. It does not depend on the size of either map. In this way, we can maintain data association in D\&C SLAM linear with the size of the joined map.

\section{EXPERIMENTS}

We have used the well-known Victoria Park dataset to validate the algorithms D\&C SLAM and RJC. This experiment is particularly adequate for testing SLAM due to its large scale and the significant level of spurious measurements. The experiment also provides critical loops in the absence of reliable features.

For RJC, we chose $b=4$, as the number of map features to be randomly selected as the seed for hypothesis generation. Two features are sufficient in theory to fix the relative location between the maps, but we have found four to adequately disambiguate. The probability that a selected feature in the overlap is not spurious, $\mathbf{P}_{\text {good }}$ is set to 0.8 , and the probability of not finding a good solution when one exists, $\mathbf{P}_{\text {fail }}$ is set to 0.01 . These parameters make the data association algorithm carry out nine random tries.

Fig. 8 shows the resulting maps from standard EKF SLAM versus D\&C SLAM. Each algorithm solves data association on its own. This allows seeing when the estimator falls out of consistency precisely because data association starts to fail;
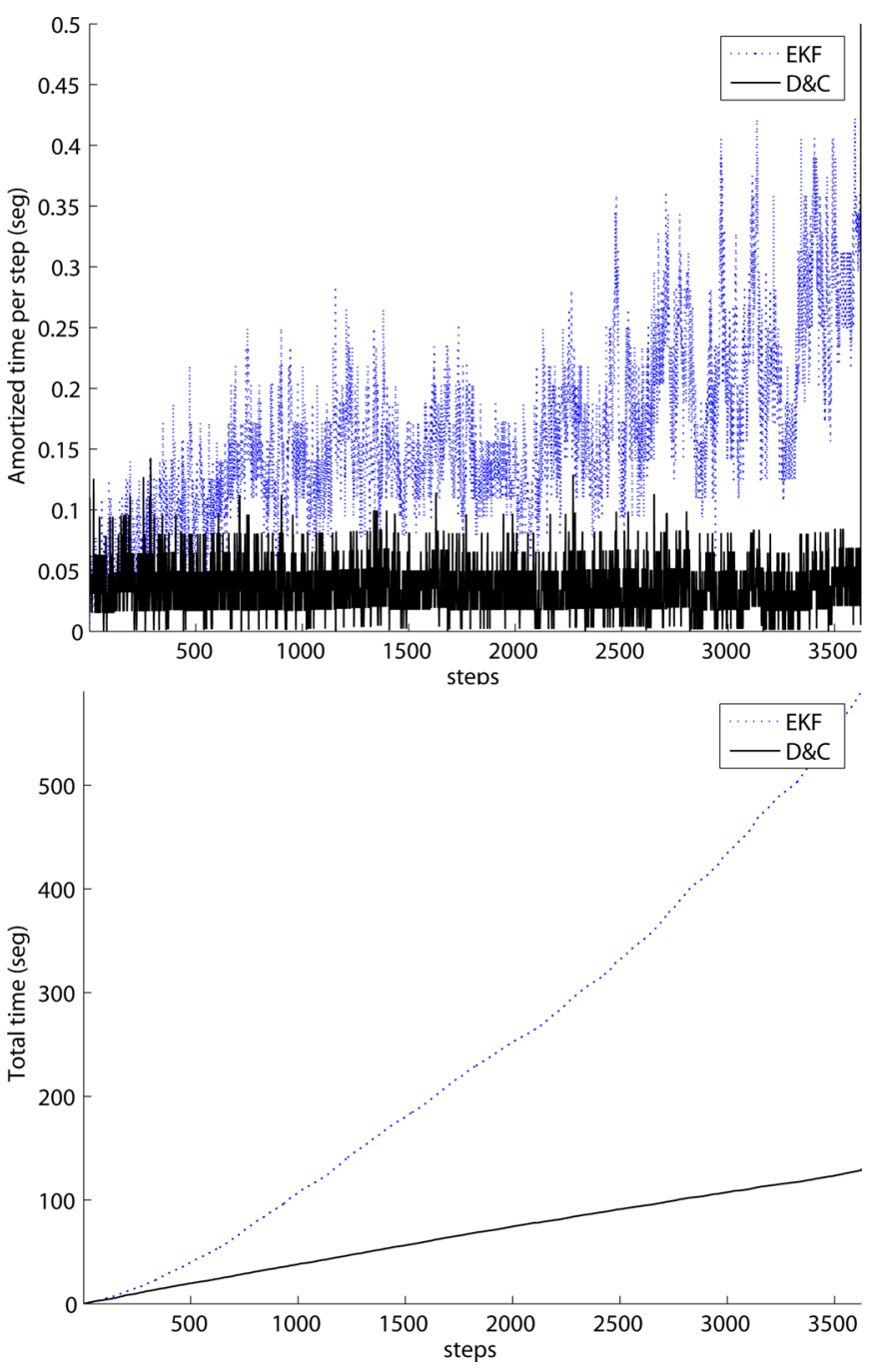

Fig. 10. (Top) Time per step of EKF SLAM versus amortized time per step of D\&C SLAM. (Bottom) Accumulated time of EKF SLAM versus D\&C SLAM

there are some minor differences due to missed associations in the case of EKF. Fig. 10 (top) shows the amortized cost of D\&C SLAM. We can see that in this experiment an EKF step can take $0.5 \mathrm{~s}$, while the amortized D\&C SLAM step will take around $0.05 \mathrm{~s}$. The main source of the noise visible in the EKF timing values is the variable number of observations gathered when the vehicle traverses the environment.

In real experiments, such as Victoria Park, it is not generally possible to predict at which step the size of the current local map will reach its limit size. This depends on the trajectory that the vehicle follows and on the density of features in the environment. Some features can be initialized in the map and later removed if they are not reobserved. In the Victoria Park dataset, the vehicle sometimes carries out exploratory trajectories, and sometimes, it revisits previously mapped regions of the park. When two local maps are joined, the size of the resulting map will depend on the overlap. For these reasons, the total map size does not increase linearly with the number of steps. This makes the total cost of standard EKF SLAM to be noncubic with the number of steps (Fig. 10, bottom). For the same reason, the total 
cost of D\&C SLAM seems to grow linearly, instead of quadratically, with the step number. In any case, the benefits of using D\&C SLAM can be clearly seen. In this experiment, the total cost of D\&C SLAM is one-fifth of the total cost of standard EKF, (130.24 s on a $2.8 \mathrm{GHz}$ Pentium IV, compared to $590.48 \mathrm{~s}$ for EKF SLAM). This result is also comparatively better than the reported by iSAM algorithm [23] (464 s on a $2 \mathrm{GHz}$ Pentium M) and comparable to FastSLAM 2.0 [29] (140 s on a $1 \mathrm{GHz}$ Pentium IV). Plotting the results of D\&C SLAM on Google Earth (see Fig. 9) reveals a superior performance when compared with the precision obtained by the mentioned algorithms [23], [29]. The accompanying video dcslam_xvid_victoria.avi shows the comparative running times of both standard EKF SLAM and D\&C SLAM.

\section{CONCLUSION}

In this paper, we have shown that EKF SLAM can be carried out in linear time with map size. We describe an EKF SLAM variant: Divide and Conquer SLAM, an algorithm that can be easily implemented. In contrast with many current efficient SLAM algorithms, all information required for data association is available when needed with no further processing. D\&C SLAM computes the EKF SLAM solution, both the state and its covariance, with no approximations, and with the additional advantage of providing always a more precise and consistent vehicle and map estimate. We also provide RJC, a data association algorithm that also executes in linear time per step.

We hope to have shown that D\&C SLAM is the algorithm to use in all applications in which the EKF solution is to be used. We also believe that the $\mathrm{D} \& \mathrm{C}$ map hierarchical splitting strategy can also be incorporated in other algorithms based on local submaps and similar strategies. This idea is part of our future research.

\section{APPENDIX}

\section{MAP JOINING 2.0}

This appendix describes the map joining process used in D\&C SLAM, an improved version with respect to the original map joining $1.0 \mathrm{in}$ [13]. The general idea is the following: in a sequential move-sense-update cycle, a local map is initialized at some moment $i$ using the current vehicle location $R_{i}$ as base reference, and thus, the initial vehicle location in the map is $\mathbf{x}_{R_{i} R_{i}}=0$ and also the initial vehicle uncertainty $\mathbf{P}_{R_{i}}=0$. Standard EKF SLAM is carried out in this move-sense-update fashion, until the map reaches a certain size of $n$ features $F_{1}, \ldots, F_{n}$ at step $j$. In this moment, the state vector $\hat{\mathbf{x}}_{i, \ldots, j}$ will be

$$
\hat{\mathbf{x}}_{i, \ldots, j}=\left[\begin{array}{c}
\hat{\mathbf{x}}_{R_{i} R_{j}} \\
\hat{\mathbf{x}}_{R_{i} F_{1}} \\
\vdots \\
\hat{\mathbf{x}}_{R_{i} F_{n}}
\end{array}\right]
$$

with corresponding covariance matrix $\mathbf{P}_{i, \ldots, j}$. This map is then closed, and a new local map $\mathbf{m}_{j, \ldots, k}=\left(\hat{\mathbf{x}}_{j, \ldots, k}, \mathbf{P}_{j, \ldots, k}\right)$ is initialized in the same way (for simplicity, assume the sensor measurements at step $j$ are used to update the first map, and the vehicle motion from $R_{j}$ to $R_{j+1}$ is carried out in the second map). This results in having the last vehicle location in the first map, $R_{j}$, be the base reference of the second map, which allows maps to be joined into a full map in a three-step process of 1) joining; 2) update; and 3) transformation, as is explained next.

\section{A. Map Joining Step}

Consider two sequential local maps $\mathbf{m}_{i, \ldots, j}=$ $\left(\hat{\mathbf{x}}_{i, \ldots, j}, \mathbf{P}_{i, \ldots, j}\right), \quad \mathbf{m}_{j, \ldots, k}=\left(\hat{\mathbf{x}}_{j, \ldots, k}, \mathbf{P}_{j, \ldots, k}\right), \quad$ with $n$ features $F_{1}, \ldots, F_{n}$ and $m$ features $G_{1}, \ldots, G_{m}$ each

$$
\hat{\mathbf{x}}_{i \ldots j}=\left[\begin{array}{c}
\mathbf{x}_{R_{i} R_{j}} \\
\mathbf{x}_{R_{i} F_{1}} \\
\vdots \\
\mathbf{x}_{R_{i} F_{n}}
\end{array}\right] ; \quad \hat{\mathbf{x}}_{j \ldots k}=\left[\begin{array}{c}
\mathbf{x}_{R_{j} R_{k}} \\
\mathbf{x}_{R_{j} G_{1}} \\
\vdots \\
\mathbf{x}_{R_{j} G_{m}}
\end{array}\right] .
$$

The joining step allows us to obtain a stochastic map $\mathbf{m}_{i, \ldots, k}^{-}=\left(\hat{\mathbf{x}}_{i, \ldots, k}^{-}, \mathbf{P}_{i, \ldots, k}^{-}\right)$in the following simple way:

$$
\hat{\mathbf{x}}_{i, \ldots, k}^{-}=\left[\begin{array}{c}
\hat{\mathbf{x}}_{i, \ldots, j} \\
\hat{\mathbf{x}}_{j, \ldots, k}
\end{array}\right] ; \quad \hat{\mathbf{P}}_{i, \ldots, k}^{-}=\left[\begin{array}{cc}
\mathbf{P}_{i \cdot \cdot j} & \mathbf{0} \\
\mathbf{0} & \mathbf{P}_{j \cdot k}
\end{array}\right] .
$$

Note that the elements in the second map are kept in their own reference $R_{j}$ instead of being referenced to reference frame $R_{i}$, as in map joining 1.0. This has the effect of delaying the linearization process of converting all features to base reference $R_{i}$ until the update step has taken place, and thus, an improved estimation is used for this linearization. This is the fundamental difference between map joining 1.0 and 2.0.

\section{B. Update Step}

Data association is carried out to determine correspondences between features coming from the first and second map. This allows us to refine the vehicle and environment feature locations by the EKF update step on the state vector. Let $\mathcal{H}$ be a hypothesis that pairs $r$ features $F_{f_{1}}, \ldots, F_{f_{r}}$ coming from local map $\mathbf{m}_{i, \ldots, j}$ with features $G_{g_{1}}, \ldots, G_{g_{r}}$ coming from map $\mathbf{m}_{j, \ldots, k}$. A modified ideal measurement equation for $r$ reobserved features expresses this coincidence

$$
\mathbf{h}_{\mathcal{H}}\left(\hat{\mathbf{x}}_{i, \ldots, k}^{-}\right)=\left[\begin{array}{c}
\mathbf{h}_{f_{1}, g_{1}} \\
\vdots \\
\mathbf{h}_{f_{r}, g_{r}}
\end{array}\right]=\mathbf{0}
$$

where, for each pairing

$$
\mathbf{h}_{f_{r}, g_{r}}=\mathbf{x}_{R_{i} F_{f_{r}}}-\mathbf{x}_{R_{i} R_{j}} \oplus \mathbf{x}_{R_{j} G_{g_{r}}} .
$$

Linearization yields

$$
\begin{aligned}
& \mathbf{h}_{\mathcal{H}}\left(\hat{\mathbf{x}}_{i, \ldots, k}^{-}\right) \simeq \mathbf{h}_{\mathcal{H}}\left(\hat{\mathbf{x}}_{i, \ldots, k}^{-}\right)+\mathbf{H}_{\mathcal{H}}\left(\mathbf{x}_{i, \ldots, k}^{-}-\hat{\mathbf{x}}_{i, \ldots, k}^{-}\right) \\
\mathbf{H}_{\mathcal{H}} & =\left.\frac{\partial \mathbf{h}_{\mathcal{H}}}{\partial \mathbf{x}_{i, \ldots, k}^{-}}\right|_{\left(\hat{\mathbf{x}}_{i, \ldots, k}^{-}\right)} \\
& =\left[\begin{array}{ccccccc}
\frac{\partial \mathbf{h}_{f_{1} g_{1}}}{\partial \mathbf{x}_{R_{i} R_{j}}} & \mathbf{0} & \cdots & \mathbf{I} & \mathbf{0} & \frac{\partial \mathbf{h}_{f_{1} g_{1}}}{\partial \mathbf{x}_{R_{j} g_{g_{1}}}} & \ldots \\
\vdots & \vdots & \vdots & \vdots & \vdots & \vdots & \vdots \\
\frac{\partial \mathbf{h}_{f g_{r}}}{\partial \mathbf{x}_{R_{i} R_{j}}} & \mathbf{0} & \mathbf{I} & \cdots & \mathbf{0} & \cdots & \frac{\partial \mathbf{h}_{f r g r}}{\partial \mathbf{x}_{R_{j} g_{g r}}}
\end{array}\right] .
\end{aligned}
$$


This article has been accepted for publication in a future issue of this journal, but has not been fully edited. Content may change prior to final publication.

The update step allows us to obtain a new estimate $\mathbf{m}_{i \ldots k}^{+}=$ $\left(\hat{\mathbf{x}}_{i, \ldots, k}^{+}, \mathbf{P}_{i, \ldots, k}^{+}\right)$by applying modified EKF update equations

$$
\begin{aligned}
\hat{\mathbf{x}}_{i, \ldots, k}^{+} & =\hat{\mathbf{x}}_{i, \ldots, k}^{-}-\mathbf{K} \mathbf{h}_{\mathcal{H}}\left(\hat{\mathbf{x}}_{i, \ldots, k}^{-}\right) \\
\mathbf{P}_{i, \ldots, k}^{+} & =\left(\mathbf{I}-\mathbf{K} \mathbf{H}_{\mathcal{H}}\right) \mathbf{P}_{i, \ldots, k}^{-} \\
\mathbf{K} & =\mathbf{P}_{i, \ldots, k}^{-} \mathbf{H}_{\mathcal{H}}^{T}\left(\mathbf{H}_{\mathcal{H}} \mathbf{P}_{i, \ldots, k}^{-} \mathbf{H}_{\mathcal{H}}^{T}\right)^{-1} .
\end{aligned}
$$

\section{Transformation Step}

A final step is carried out to transform all the elements of $\hat{\mathbf{x}}_{i, \ldots, k}^{+}$to the same base reference $R_{i}$ and obtain the final joined map $\mathbf{m}_{i, \ldots, k}=\left(\hat{\mathbf{x}}_{i, \ldots, k}, \mathbf{P}_{i, \ldots, k}\right)$

$$
\begin{aligned}
& \hat{\mathbf{x}}_{i, \ldots, k}=\left[\begin{array}{c}
\hat{\mathbf{x}}_{R_{i} R_{k}} \\
\hat{\mathbf{x}}_{R_{i} F_{1}} \\
\vdots \\
\hat{\mathbf{x}}_{R_{i} F_{n}} \\
\hat{\mathbf{x}}_{R_{i} G_{1}} \\
\vdots \\
\hat{\mathbf{x}}_{R_{i} G_{m}}
\end{array}\right]=\left[\begin{array}{c}
\hat{\mathbf{x}}_{R_{i} R_{j}}^{+} \oplus \hat{\mathbf{x}}_{R_{j} R_{k}}^{+} \\
\hat{\mathbf{x}}_{R_{i} F_{1}}^{+} \\
\vdots \\
\hat{\mathbf{x}}_{R_{i} F_{n}}^{+} \\
\hat{\mathbf{x}}_{R_{i} R_{j}}^{+} \oplus \hat{\mathbf{x}}_{R_{j} G_{1}}^{+} \\
\vdots \\
\hat{\mathbf{x}}_{R_{i} R_{j}}^{+} \oplus \hat{\mathbf{x}}_{R_{j} G_{m}}^{+}
\end{array}\right] \\
& \mathbf{P}_{i, \ldots, k}=\frac{\partial \hat{\mathbf{x}}_{i, \ldots, k}}{\partial \hat{\mathbf{x}}_{i, \ldots, k}^{+}} \mathbf{P}_{i, \ldots, k}^{+}\left(\frac{\partial \hat{\mathbf{x}}_{i, \ldots, k}}{\partial \hat{\mathbf{x}}_{i, \ldots, k}^{+}}\right)^{T} \\
& \frac{\partial \hat{\mathbf{x}}_{i, \ldots, k}}{\partial \hat{\mathbf{x}}_{i, \ldots, k}^{+}}=\left[\begin{array}{cccc}
\frac{\partial \mathbf{x}_{R_{i} R_{k}}}{\partial \mathbf{x}_{R_{i} R_{j}}} & \mathbf{0} & \frac{\partial \mathbf{x}_{R_{i} R_{k}}}{\partial \mathbf{x}_{R_{j} R_{k}}} & \mathbf{0} \\
\mathbf{0} & \mathbf{I} & \mathbf{0} & \mathbf{0} \\
\frac{\partial \mathbf{x}_{R_{i} E}}{\partial \mathbf{x}_{R_{i} R_{j}}} & \mathbf{0} & \mathbf{0} & \frac{\partial \mathbf{x}_{R_{i} E}}{\partial \mathbf{x}_{R_{j} E}}
\end{array}\right] .
\end{aligned}
$$

Again note that this linearization is carried out once the map has been refined in the previous update step, thus using a better estimate.

\section{REFERENCES}

[1] L. M. Paz, P. Jensfelt, J. D. Tardós, and J. Neira, "EKF SLAM updates in $O(n)$ with divide and conquer SLAM," in Proc. IEEE Int. Conf. Robot. Autom., Rome, Italy, Apr. 2007, pp. 1657-1663.

[2] L. M. Paz, J. Guivant, J. D. Tardós, and J. Neira, "Data association in $O(n)$ for divide and conquer SLAM," presented at the Robot. Sci. Syst. Conf., Atlanta, GA, Jun. 2007.

[3] H. Durrant-Whyte and T. Bailey, "Simultaneous localization and mapping: Part I," IEEE Robot. Autom. Mag., vol. 13, no. 2, pp. 99-110, Jun. 2006.

[4] T. Bailey and H. Durrant-Whyte, "Simultaneous localization and mapping (SLAM): Part II," IEEE Robot. Autom. Mag., vol. 13, no. 3, pp. 108-117, Sep. 2006.

[5] R. Chatila and J. Laumond, "Position referencing and consistent world modeling for mobile robots," in Proc. IEEE Int. Conf. Robot. Autom., 1985, vol. 2, pp. 138-145.

[6] R. C. Smith and P. Cheeseman, "On the representation and estimation of spatial uncertainty," Int. J. Robot. Res., vol. 5, no. 4, pp. 56-68, 1986.

[7] R. Smith, M. Self, and P. Cheeseman, "A Stochastic map for uncertain Spatial relationships," in Robotics Res., 4th Int. Symp., O. Faugeras and G. Giralt, Eds. Cambridge, MA: MIT Press, 1988, pp. 467-474.

[8] J. Leonard and H. Durrant-Whyte, "Simultaneous map building and localization for an autonomous mobile robot," in Proc. IEEE/RJS Int. Conf. Intell. Robots Syst., Osaka, Japan, 1991, pp. 1442-1447.

[9] J. A. Castellanos and J. D. Tardós, Mobile Robot Localization and Map Building: A Multisensor Fusion Approach. Boston, MA: Kluwer, 1999.

[10] J. E. Guivant and E. M. Nebot, "Optimization of the simultaneous localization and map-building algorithm for real-time implementation," IEEE Trans. Robot. Autom., vol. 17, no. 3, pp. 242-257, May 2001.
[11] J. Knight, A. Davison, and I. Reid, "Toward constant time SLAM using postponement," in Proc. IEEE/RSJ Int. Conf. Intell. Robots Syst., Maui, HI, 2001, pp. 406-412.

[12] S. J. Julier, "A Sparse weight kalman filter approach to simultaneous localization and map building," in Proc. IEEE/RSJ Int. Conf. Intell. Robots Syst., Maui, HI, Oct. 2001, vol. 1, pp. 1251-1256.

[13] J. D. Tardós, J. Neira, P. M. Newman, and J. J. Leonard, "Robust mapping and localization in indoor environments using Sonar data," Int. J. Robot. Res., vol. 21, no. 4, pp. 311-330, 2002.

[14] L. M. Paz and J. Neira, "Optimal local map size for EKF-based SLAM," in Proc. IEEE/RSJ Int. Conf. Intell. Robots Syst., Beijing, China, Oct. 2006, pp. 5019-5025.

[15] S. Thrun, Y. Liu, D. Koller, A. Y. Ng, Z. Ghahramani, and H. DurrantWhyte, "Simultaneous localization and mapping with sparse extended information filters," Int. J. Robot. Res., vol. 23, no. 7/8, pp. 693-716, 2004.

[16] R. Eustice, M. Walter, and J. Leonard, "Sparse extended information filters: insights into sparsification," in Proc. IEEE/RSJ Int. Conf. Intell. Robots Syst., Edmonton, AB, Canada, Aug. 2005, pp. 3281-3288.

[17] M. R. Walter, R. M. Eustice, and J. J. Leonard, "Exactly sparse extended information filters for feature-based SLAM," Int. J. Robot. Res., vol. 26 , no. 4, pp. 335-359, 2007.

[18] M. A. Paskin, "Thin junction tree filters for simultaneous localization and mapping," in Proc. Int. Joint Conf. Artif. Intell., San Francisco, CA, 2003, pp. 1157-1164.

[19] U. Frese, "Treemap: an $\mathrm{O}(\log \mathrm{n})$ algorithm for indoor simultaneous localization and mapping," Auton. Robots, vol. 21, no. 2, pp. 103-122, Sep. 2006.

[20] R. M. Eustice, H. Singh, and J. J. Leonard, "Exactly sparse delayedstate filters for view-based SLAM," IEEE Trans. Robot., vol. 22, no. 6 , pp. 1100-1114, Dec. 2006.

[21] F. Dellaert and M. Kaess, "square root SAM: Simultaneous localization and mapping via square root information smoothing," Int. J. Robot. Res., vol. 25 , no. 12 , pp. 1181-1203, Dec. 2006.

[22] K. Ni, D. Steedly, and F. Dellaert, "Tectonic SAM: exact, out-of-core, submap-based SLAM," in Proc. IEEE Int. Conf. Robot. Autom., Rome, Italy, Apr. 2007, pp. 1678-1685.

[23] M. Kaess, A. Ranganathan, and F. Dellaert, "iSAM: fast incremental smoothing and mapping with efficient data association," in Proc. IEEE Int. Conf. Robot. Autom. (ICRA), Rome, Italy, Apr. 2007, pp. 16701677.

[24] S. J. Julier and J. K. Uhlmann, "A counter example to the theory of simultaneous localization and map building," in Proc. IEEE Int. Conf. Robot. Autom., Seoul, Korea, 2001, pp. 4238-4243.

[25] J. A. Castellanos, J. Neira, and J. D. Tardós, "Limits to the consistency of EKF-based SLAM," presented at the 5th IFAC Symp. Intell. Auton. Vehicles, Lisbon, Portugal, 2004.

[26] T. Bailey, J. Nieto, J. Guivant, M. Stevens, and E. Nebot, "Consistency of the EKF-SLAM algorithm," in Proc. IEEE/RJS Int. Conf. Intell. Robots Syst., Beijing, China, 2006, pp. 3562-3568.

[27] S. Julier and J. Uhlmann, "A new extension of the kalman filter to nonlinear systems," in Proc. Int. Symp. Aerosp./Defense Sens., Simulate Controls, Orlando, FL, 1997, pp. 182-193.

[28] R. Martinez-Cantin and J. A. Castellanos, "Unscented SLAM for largescale outdoor environments," in Proc. IEEE/RSJ Int. Conf. Intell. Robots Syst., Edmonton, AB, Canada, 2005, pp. 328-333.

[29] M. Montemerlo, S. Thrun, D. Koller, and B. Wegbreit, "Fastslam 2.0: an improved particle filtering algorithm for simultaneous localization and mapping that provably converges," in Proc. Int. Joint Conf. Artif. Intell., 2003, pp. 1151-1156.

[30] S. Huang and G. Dissanayake, "Convergence and consistency analysis for extended Kalman filter-based SLAM," IEEE Trans. Robot., vol. 23, no. 5, pp. 1036-1049, Oct. 2007

[31] M. W. M. G. Dissanayake, P. Newman, S. Clark, H. F. Durrant-Whyte, and M. Csorba, "A solution to the simultaneous localization and map building (SLAM) problem," IEEE Trans. Robot. Autom., vol. 17, no. 3, pp. 229-241, Jun. 2001.

[32] J. Leonard and H. Feder, "Decoupled stochastic mapping," IEEE J. Ocean. Eng., vol. 26, no. 4, pp. 561-571, Oct. 2001.

[33] J. Leonard and P. Newman, "Consistent, convergent, and constant-time SLAM," in Proc. Int. Joint Conf. Artif. Intell., Acapulco, Mexico, Aug. 2003, pp. 1143-1150.

[34] M. Bosse, P. M. Newman, J. J. Leonard, and S. Teller, "SLAM in largescale cyclic environments using the atlas framework," Int. J. Robot. Res. vol. 23, no. 12, pp. 1113-1139, Dec. 2004. 
This article has been accepted for publication in a future issue of this journal, but has not been fully edited. Content may change prior to final publication.

[35] C. Estrada, J. Neira, and J. D. Tardós, "Hierarchical SLAM: Real-time accurate mapping of large environments," IEEE Trans. Robot., vol. 21, no. 4, pp. 588-596, Aug. 2005.

[36] S. B. Williams, "Efficient solutions to autonomous mapping and navigation problems," Ph.D. dissertation, Australian Centre Field Robot., Univ. Sydney, Sydney, Australia, Sep. 2001.

[37] W. E. L. Grimson, Object Recognition by Computer: The Role of Geometric Constraints. Cambridge, MA: MIT Press, 1990.

[38] Y. Bar-Shalom and T. E. Fortmann, Tracking and Data Association. New York: Academic, 1988.

[39] J. Neira and J. D. Tardós, "Data association in Stochastic mapping using the joint compatibility test," IEEE Trans. Robot. Autom., vol. 17, no. 6, pp. 890-897, Dec. 2001.

[40] L. Clemente, A. J. Davison, I. D. Reid, J. Neira, and J. D. Tardós, "Mapping large loops with a single hand-held camera," presented at the Robot. Sci. Syst. Conf., Atlanta, GA, Jun. 2007.

[41] J. Uhlmann, "Introduction to the algorithmics of data association in multiple-target tracking," in Handbook of Multisensor Data Fusion. Boca Raton, FL: CRC, 2001.

[42] J. Neira, J. D. Tardós, and J. A. Castellanos, "Linear time vehicle relocation in SLAM," in Proc. IEEE Int. Conf. Robot. Autom., Taipei, Taiwan, R.O.C., Sep. 2003, pp. 427-433.

[43] R. Hartley and A. Zisserman, Multiple View Geometry in Computer Vision. Cambridge, U.K.: Cambridge Univ. Press, 2000.

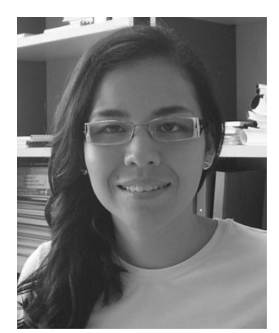

Lina M. Paz (M’08) was born in Cali, Colombia, in 1980. She received the M.S. degree in electronic engineering from the Universidad del Valle, Cali, in 2003. She is currently working toward the Ph.D. degree in computer science with the Department of Computer Science and Systems Engineering, University of Zaragoza, Zaragoza, Spain.

Her current research interests include mobile robotics, computer vision for environment modeling, and simultaneous localization and mapping (SLAM).

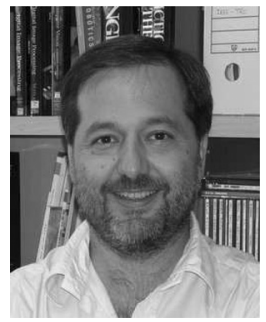

Juan D. Tardós (M'05) was born in Huesca, Spain, in 1961. He received the M.S. and Ph.D. degrees in electrical engineering from the University of Zaragoza, Zaragoza, Spain, in 1985 and 1991, respectively.

He is currently a Full Professor with the Department of Computer Science and Systems Engineering, University of Zaragoza, where he is in charge of courses in robotics, computer vision, and artificial intelligence. His current research interests include simultaneous localization and mapping (SLAM) and perception and mobile robotics.

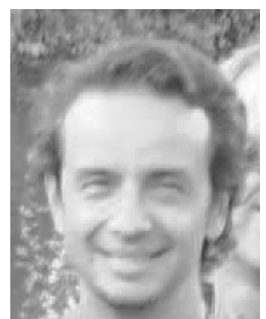

José Neira (M'07) was born in Bogotá, Colombia, in 1963. He received the M.S. degree from the Universidad de los Andes, Bogotá, and the Ph.D. degree from the University of Zaragoza, Zaragoza, Spain, in 1986 and 1993, respectively, both in computer science.

$\mathrm{He}$ is currently an Associate Professor with the Department of Computer Science and Systems Engineering, University of Zaragoza, where he teaches compiler theory, computer vision, and mobile robotics. His current research interests include autonomous robots, data association, and environment modeling. 